\title{
Revealed preference and choice under uncertainty
}

\author{
Walter Bossert • Kotaro Suzumura
}

Received: 6 November 2010 / Accepted: 29 January 2011 / Published online: 24 February 2011 (C) The Author(s) 2011. This article is published with open access at SpringerLink.com

\begin{abstract}
We employ the theory of rational choice to examine whether observable choices from feasible sets of prospects can be generated by the optimization of some underlying decision criterion under uncertainty. Rather than focusing on a specific theory of choice, our objective is to formulate a general approach that is designed to cover the various decision criteria that have been proposed in the literature. We use a mild dominance property to define a class of suitable choice criteria. In addition to rationalizability per se, we characterize transitive and Suzumura consistent rationalizability in the presence of dominance.
\end{abstract}

Keywords Uncertainty $\cdot$ Prospects $\cdot$ Rational choice $\cdot$ Decision theory

JEL Classification D11 D D 1

This paper is dedicated to Salvador Barberà in appreciation of his invaluable contribution to the academic community in general and to the group of economic theorists in particular. We thank a referee for her or his extremely thoughtful comments and suggestions. Financial support from a Grant-in-Aid for Specially Promoted Research from the Ministry of Education, Culture, Sports, Science and Technology of Japan for the Project on Economic Analysis of Intergenerational Issues (grant number 22000001), the Fonds de Recherche sur la Société et la Culture of Québec and the Social Sciences and Humanities Research Council of Canada is gratefully acknowledged.

W. Bossert $(\varangle)$

Department of Economics and CIREQ, University of Montreal, P.O. Box 6128, Station Downtown, Montreal, QC, H3C 3J7, Canada

e-mail: walter.bossert@umontreal.ca

K. Suzumura

School of Political Science and Economics, Waseda University, 1-6-1 Nishi-Waseda, Shinjuku-ku, Tokyo 169-8050, Japan

e-mail: k.suzumura@aoni.waseda.jp 


\section{Introduction}

It is by now well-established that the choice behavior of an economic agent can be considered more fundamental than the optimization of some objective. An agent's choices can be observed directly and, recognizing this early on in the development of demand analysis, Samuelson $(1938,1948)$ laid the foundations of what has come to be known as revealed preference theory; see also Houthakker (1950), among others. A consumer's demand function is taken as the primitive of the problem and the basic question is whether the observable behavior of this agent is consistent with the standard hypothesis of utility maximization under budget constraints. Although these early contributions restricted attention to consumer choice in perfectly competitive markets, the theory of rational choice progressed rapidly and more general choice scenarios were analyzed in contributions such as those of Richter (1966), Hansson (1968) and Suzumura (1976a, 1977). Initially, the analysis of rational choice behavior focused on models where a rationalizing relation was assumed to be an ordering but, more recently, weaker coherence properties of a rationalization have been considered; see, for instance, Richter (1971) for an early contribution in this spirit. A detailed review of rational choice and revealed preference theory can be found in Bossert and Suzumura (2010).

Numerous theories of choice under uncertainty have emerged over the years; prominent examples include proposals by von Neumann and Morgenstern (1944), Milnor (1954), Savage (1954), Barberà et al. (1984), Barberà and Pattanaik (1984) and Kannai and Peleg (1984). Both probabilistic and non-probabilistic choice models (such as setbased models) are covered by these and other contributions. In this paper, we aim at combining the theory of rational choice with a general approach to choice situations under uncertainty.

In decision problems under certainty, the revealed preference approach typically does not try to uncover a specific objective the optimization of which may be revealed through the observed choices; rather, the fundamental question is whether these choices are consistent with some objective in the sense that the choices from each feasible set are the greatest elements according to a rationalizing relation. Analogously, we do not want to restrict ourselves to a specific theory of choice under uncertainty. Our basic question is whether observable choices can be consistent with some coherent way of making decisions in the presence of uncertainty. We therefore use as our primitive a set of prospects. Suppose there is a universal set $X$ (with at least two members) of certain outcomes and a finite number of (at least two) possible states of the world. A prospect assigns to each possible state an outcome in $X$. We can think of a prospect as the result of an action taken by an agent before the uncertainty regarding the state that actually occurs is resolved. The reason why we choose prospects to provide a fundamental description of our choice situations is the generality we aim to achieve: if we were, for instance, to use lotteries as the objects to be chosen from, we would already be committed to a probabilistic choice model. Because we do not want to rule out non-probabilistic models such as those examined by Milnor (1954) and Barberà and Pattanaik (1984), for instance, we choose to use prospects as our basic representation of choice situations under uncertainty. However, models that do endow a decision maker with a probability distribution are included as special cases in our approach. 
The definition of a decision rule that we use in this paper is based on what we think is a minimal requirement. Given a choice function defined on a domain of sets of feasible prospects, we first demand that there be a relation that rationalizes the observable choices in the usual sense of generating them as greatest elements in the requisite feasible set. In addition, we ask that the rationalization satisfy a dominance property so as to be interpretable as a choice rule under uncertainty. The dominance condition is easily described: if two prospects $\mathbf{x}=\left(x_{1}, \ldots, x_{m}\right)$ and $\mathbf{y}=\left(y_{1}, \ldots, y_{m}\right)$ are such that, for every state $i$ and for every state $j$, the certain prospect $\left(x_{i}, \ldots, x_{i}\right)$ that repeats outcome $x_{i}$ in $\mathbf{x}$ over all states is revealed to be at least as good as the certain prospect $\left(y_{j}, \ldots, y_{j}\right)$ that repeats outcome $y_{j}$ in $\mathbf{y}$ over all states, then prospect $\mathbf{x}$ must be at least as good as prospect $\mathbf{y}$. This is a very weak requirement because we do not demand state-by-state dominance to be respected but, instead, merely unambiguous dominance where the worst possible certain outcome in $\mathbf{x}$ is at least as good at the best possible certain outcome in $\mathbf{y}$. Although this condition that we impose in addition to rationalizability per se is rather mild, it does impose further restrictions, as is shown once our formal framework is introduced.

There is a resemblance to the analysis carried out by Bossert (2001) who also examines rationalizability in the context of uncertainty. However, Bossert (2001) restricts attention to set-based models whereas our approach is considerably more general. Because of the more specialized framework, Bossert's (2001) restrictions differ from our dominance property.

In the next section, we define the fundamentals of the problem to be addressed in this paper, namely, the notions of prospects and choice functions. Section 3 provides necessary and sufficient conditions for dominance rationalizability (that is, rationalizability by a relation that respects the above-described dominance requirement). In Sects. 4 and 5 , we add the coherence properties of transitivity and of Suzumura consistency, respectively, to the list of requirements imposed on a rationalization. Section 6 concludes.

\section{Prospects and choice functions}

Suppose there is a set of alternatives or outcomes $X$ with at least two elements. $X$ could be finite or infinite. There are $m \in \mathbb{N} \backslash\{1\}$ possible states of the world and a prospect $\mathbf{x}=\left(x_{1}, \ldots, x_{m}\right)$ with $x_{i} \in X$ for all $i \in\{1, \ldots, m\}$ specifies, for each state, the alternative that materializes in this state. The set of all prospects is denoted by $X^{m}$. Important special cases of prospects are the certain prospects in which the same outcome emerges in all possible states. For any $x \in X$, the certain prospect associated with alternative $x$ is the $m$-tuple $(x, \ldots, x)$ and we will denote this $m$-tuple by $x \mathbf{1}_{m}$. The set of all non-empty subsets of $X^{m}$ is $\mathcal{X}$.

A (binary) relation on $X^{m}$ is a subset $R$ of the Cartesian product $X^{m} \times X^{m}$ and the asymmetric part of $R$ is $P(R)$. The transitive closure tc $(R)$ of a relation $R$ on $X^{m}$ is

$$
\begin{aligned}
\operatorname{tc}(R)= & \left\{(\mathbf{x}, \mathbf{y}) \mid \text { there exist } K \in \mathbb{N} \text { and } \mathbf{x}^{0}, \ldots, \mathbf{x}^{K} \in X^{m}\right. \text { such that } \\
& {\left.\left[\mathbf{x}=\mathbf{x}^{0} \text { and }\left(\mathbf{x}^{k-1}, \mathbf{x}^{k}\right) \in R \text { for all } k \in\{1, \ldots, K\} \text { and } \mathbf{x}^{K}=\mathbf{y}\right]\right\} . }
\end{aligned}
$$

The transitive closure of a relation $R$ is the smallest transitive relation containing $R$. 
A relation $R$ is Suzumura consistent if and only if, for all $K \in \mathbb{N}$ and for all $\mathbf{x}, \mathbf{y} \in X^{m}$,

$$
(\mathbf{x}, \mathbf{y}) \in t c(R) \Rightarrow(\mathbf{y}, \mathbf{x}) \notin P(R)
$$

The Suzumura consistent closure $\operatorname{sc}(R)$ of a relation $R$ is given by

$$
s c(R)=R \cup\{(\mathbf{x}, \mathbf{y}) \mid(\mathbf{x}, \mathbf{y}) \in t c(R) \text { and }(\mathbf{y}, \mathbf{x}) \in R\} .
$$

Suzumura consistency was first introduced in Suzumura (1976b). Analogous to the transitive closure of a relation, the Suzumura consistent closure of $R$ is the smallest Suzumura consistent relation containing $R$. The notion of a Suzumura consistent closure is due to Bossert et al. (2005). See Bossert and Suzumura (2010) for a detailed discussion of Suzumura consistency and its use in individual and collective choice.

We assume that, in the presence of uncertainty, a decision maker faces a set of feasible actions and that each action leads to a prospect in $X^{m}$. Rather than working with actions and their induced prospects, we work with prospects directly in order to simplify our exposition. Thus, a choice rule under uncertainty can be expressed by means of a choice function that selects, from each feasible set of prospects in its domain, a non-empty subset of this feasible set. The only assumption (other than non-emptiness) that we make about the domain $\Sigma$ of a choice function is that it includes all singletons and pairs of certain prospects. Formally, a choice function with a certainty inclusive domain is a mapping $C: \Sigma \rightarrow \mathcal{X}$ such that $\left\{\left\{x \mathbf{1}_{m}, y \mathbf{1}_{m}\right\} \mid x, y \in X\right\} \subseteq \Sigma \subseteq \mathcal{X}$ and $C(S) \subseteq S$ for all $S \in \Sigma$. Because the certainty inclusiveness assumption will be maintained throughout the paper, we will simply refer to $C$ as a choice function with the understanding that this function has a certainty inclusive domain.

\section{Dominance rationalizability}

As is well-known, a choice function $C$ is rationalizable by a binary relation $R$ on $X^{m}$ if and only if, for each feasible set of prospects $S$ in the domain $\Sigma, C$ selects the $R$-greatest elements in $S$. However, in the present context of choice under uncertainty, we might want to impose more than just the standard rationalizability property in order to think of a choice function as representing a plausible method of selecting from sets of available prospects. Clearly, there are many theories of choice under uncertainty such as those pioneered and discussed by von Neumann and Morgenstern (1944), Milnor (1954), Savage (1954), Fishburn (1970), Arrow and Hurwicz (1972), Gärdenfors (1976), Kim and Roush (1980), Barberà et al. (1984), Barberà and Pattanaik (1984), Kannai and Peleg (1984), Barberà et al. (2004), to name but a few.

The main purpose of the approach advocated in this paper is not the identification of a specific theory of choice under uncertainty but, rather, to define a more general criterion that subsumes many of the models proposed so far. Clearly, this means that our definition of possible choice rules is quite permissive. In addition to probabilistic choice rules such as those proposed by von Neumann and Morgenstern (1944) or Savage (1954), our class of choice rules includes many others that are not based on 
(objective or subjective) probabilities, such as those discussed by Milnor (1954) or Kannai and Peleg (1984), for instance. Of course, our definition of possible choice rules still has some bite in that it allows us to eliminate rules that we consider unacceptable given our interpretation; this is illustrated below via a simple example. As we discuss in the concluding section, our approach can be amended in a straightforward and intuitive manner if one desires to come up with a more stringent definition. What we think of as the major contribution of this paper is the method we propose to incorporate notions of uncertainty into a model of rational choice.

Coming back to our definition of rationalizability in the current context, we propose as a minimal requirement that, in addition to rationalizability per se, a weak dominance property be respected. More precisely, we demand that observed choices involving certain prospects be respected in the following sense. Consider two prospects $\mathbf{x}=\left(x_{1}, \ldots, x_{m}\right)$ and $\mathbf{y}=\left(y_{1}, \ldots, y_{m}\right)$. If every certain prospect $x_{i} \mathbf{1}_{m}$ with $i \in\{1, \ldots, m\}$ is revealed to be weakly preferred to every certain prospect $y_{j} \mathbf{1}_{m}$ with $j \in\{1, \ldots, m\}$ in the sense that $x_{i} \mathbf{1}_{m}$ is chosen in a situation where $y_{j} \mathbf{1}_{m}$ is feasible, then the relation rationalizing $C$ must declare $\mathbf{x}$ to be at least as good as $\mathbf{y}$. Formally, we say that a choice function $C$ is dominance rationalizable if and only if there exists a relation $R$ on $X^{m}$ such that

$$
C(S)=\{\mathbf{x} \in S \mid(\mathbf{x}, \mathbf{y}) \in R \text { for all } \mathbf{y} \in S\} \quad \text { for all } S \in \Sigma
$$

and

$$
\left[\left(x_{i} \mathbf{1}_{m}, y_{j} \mathbf{1}_{m}\right) \in R \text { for all } i, j \in\{1, \ldots, m\} \Rightarrow(\mathbf{x}, \mathbf{y}) \in R\right] \quad \text { for all } \mathbf{x}, \mathbf{y} \in X^{m}
$$

If $C$ and $R$ are such that (1) and (2) are satisfied, we also say that $R$ is a dominance rationalization of $C$ or that $C$ is dominance rationalized by $R$.

Property (1) represents the standard rationalizability requirement: for any feasible set $S$ in the domain of a choice function $C$, the set of chosen elements $C(S)$ must coincide with the set of $R$-greatest elements in $S$ according to a (dominance) rationalization $R$.

That (2) imposes additional restrictions on $C$ can be seen by considering the following example. Suppose that the set of certain alternatives is $X=\{x, y, z\}$, that there are $m=2$ possible states of the world, and the certainty inclusive domain of $C$ is given by

$$
\begin{aligned}
\Sigma= & \{\{(x, x)\},\{(y, y)\},\{(z, z)\}, \\
& \{(x, x),(y, y)\},\{(x, x),(z, z)\},\{(y, y),(z, z)\}, \\
& \{(x, y),(y, z)\}\} .
\end{aligned}
$$

Now define the choice function $C$ by letting

$$
\begin{aligned}
& C(\{(x, x)\})=\{(x, x)\}, C(\{(y, y)\})=\{(y, y)\}, C(\{(z, z)\})=\{(z, z)\}, \\
& C(\{(x, x),(y, y)\})=\{(x, x)\}, C(\{(x, x),(z, z)\})=\{(x, x)\}, C(\{(y, y),(z, z)\})=\{(y, y)\}, \\
& C(\{(x, y),(y, z)\})=\{(y, z)\} .
\end{aligned}
$$


Consider the relation $R$ on $X^{m}$ defined by

$$
\begin{aligned}
R= & \{((x, x),(x, x)),((y, y),(y, y)),((z, z),(z, z)), \\
& ((x, x),(y, y)),((x, x),(z, z)),((y, y),(z, z)), \\
& ((y, z),(x, y))\} .
\end{aligned}
$$

It is straightforward to verify that (1) is satisfied for $C$ and $R$. However, there exists no relation $R^{\prime}$ such that, for $C$ and $R^{\prime}$, (2) is satisfied in addition to (1). By way of contradiction, suppose $R^{\prime}$ is such a relation. First of all, as a consequence of (1) and the definition of $C$, we must have $((x, x),(y, y)) \in R^{\prime},((y, y),(y, y)) \in R^{\prime}$, $((x, x),(z, z)) \in R^{\prime}$ and $((y, y),(z, z)) \in R^{\prime}$. Thus, (2) implies $((x, y),(y, z)) \in R^{\prime}$. Therefore, the prospect $(x, y)$ is an $R^{\prime}$-greatest element in $\{(x, y),(y, z)\}$ and (1) demands that $(x, y) \in C(\{(x, y),(y, z)\})$, in contradiction to the definition of $C$. Thus, even though the additional property that we require of a rationalization in the context of choice under uncertainty is very weak, it is not redundant and can be used to eliminate rules that are in violation of the basic dominance condition (2).

Richter (1971) characterizes rational choice in a general setting where no additional requirements such as that expressed by (2) are imposed. In our framework, an analogous result can be obtained by modifying his necessary and sufficient condition in a suitable manner. To do so, we first introduce the notion of the direct revealed preference relation $R_{C}^{d}$ associated with a choice function $C$. This relation is defined by letting, for all $\mathbf{x}, \mathbf{y} \in X^{m}$,

$$
(\mathbf{x}, \mathbf{y}) \in R_{C}^{d} \Leftrightarrow \text { there exists } S \in \Sigma \text { such that }[\mathbf{x} \in C(S) \text { and } \mathbf{y} \in S] \text {. }
$$

Because we have to take into account the dominance property in addition to mere rationalizability, we consider the following relation that incorporates this requirement. The direct revealed preference and dominance relation $R_{C}$ corresponding to $C$ is defined by letting, for all $\mathbf{x}, \mathbf{y} \in X^{m}$,

$$
(\mathbf{x}, \mathbf{y}) \in R_{C} \Leftrightarrow(\mathbf{x}, \mathbf{y}) \in R_{C}^{d} \text { or }\left[\left(x_{i} \mathbf{1}_{m}, y_{j} \mathbf{1}_{m}\right) \in R_{C}^{d} \text { for all } i, j \in\{1, \ldots, m\}\right] .
$$

Following Samuelson's $(1938,1948)$ observation in the context of rationality in consumer choice problems, Richter (1971) establishes that the direct revealed preference relation $R_{C}^{d}$ associated with a choice function $C$ must be respected by any rationalizing relation $R$ in the sense that $R_{C}^{d}$ is contained in $R$. An analogous result is valid in our setting. However, because of the additional dominance requirement we impose, the relation $R_{C}$ rather than $R_{C}^{d}$ must be respected when choices are made from sets of feasible prospects. This leads to the following result, which is analogous to the above-mentioned observation due to Richter (1971).

Lemma 1 If a choice function $C$ is dominance rationalized by a relation $R$, then $R_{C} \subseteq R$.

Proof Suppose that $R$ is a dominance rationalization of $C$ and that $(\mathbf{x}, \mathbf{y}) \in R_{C}$. By definition of $R_{C}$, there are two possible cases: 
(a) $(\mathbf{x}, \mathbf{y}) \in R_{C}^{d}$;

(b) $\left(x_{i} \mathbf{1}_{m}, y_{j} \mathbf{1}_{m}\right) \in R_{C}^{d}$ for all $i, j \in\{1, \ldots, m\}$.

In case (a), the definition of $R_{C}^{d}$ implies that there exists $S \in \Sigma$ such that $\mathbf{x} \in C(S)$ and $\mathbf{y} \in S$. Thus, $\mathbf{x}$ is an $R$-greatest element in $S$ by (1) which, together with $\mathbf{y} \in S$, implies $(\mathbf{x}, \mathbf{y}) \in R$.

In case (b), the result just established for case (a) implies that $\left(x_{i} \mathbf{1}_{m}, y_{j} \mathbf{1}_{m}\right) \in R$ for all $i, j \in\{1, \ldots, m\}$. By (2), it follows that $(\mathbf{x}, \mathbf{y}) \in R$.

We can now use this lemma to characterize dominance rationalizability. Again, the method of proof is based on that employed by Richter (1971). In our framework, however, some additional steps are needed as a consequence of imposing the dominance requirement. The following property of a choice function $C$ turns out to be necessary and sufficient for dominance rationalizability.

Direct dominance revelation coherence. For all $S \in \Sigma$ and for all $\mathbf{x} \in X^{m}$,

$$
(\mathbf{x}, \mathbf{y}) \in R_{C} \text { for all } \mathbf{y} \in S \Rightarrow \mathbf{x} \in C(S)
$$

Direct dominance revelation coherence requires that the relation $R_{C}$ be respected by the choice function $C$. It is relatively straightforward to see that this is indeed necessary for dominance rationalizability. As established in the following theorem, the property is also sufficient.

Theorem 1 A choice function $C$ is dominance rationalizable if and only if $C$ satisfies direct dominance revelation coherence.

Proof We first prove the only if part of the equivalence stated in the theorem. Suppose $R$ is a dominance rationalization of $C$. Let $S \in \Sigma$ and $\mathbf{x} \in S$ be such that $(\mathbf{x}, \mathbf{y}) \in R_{C}$ for all $\mathbf{y} \in S$. By Lemma 1 , it follows that $(\mathbf{x}, \mathbf{y}) \in R$ for all $\mathbf{y} \in S$. Because $R$ is a dominance rationalization of $C$, this implies $\mathbf{x} \in C(S)$ and direct dominance revelation coherence is established.

To prove the if part of the theorem, suppose that $C$ satisfies direct dominance revelation coherence. We now show that $R=R_{C}$ is a dominance rationalization of $C$.

To establish that (1) is satisfied for $R=R_{C}$, suppose first that $S \in \Sigma$ and $\mathbf{x} \in S$ are such that $(\mathbf{x}, \mathbf{y}) \in R_{C}$ for all $\mathbf{y} \in S$. Direct dominance revelation coherence immediately implies $\mathbf{x} \in C(S)$.

Now suppose that $S \in \Sigma$ and $\mathbf{x} \in S$ are such that $\mathbf{x} \in C(S)$. By definition, this implies $(\mathbf{x}, \mathbf{y}) \in R_{C}^{d}$ for all $\mathbf{y} \in S$ and, because $R_{C}^{d} \subseteq R_{C}$, we obtain $(\mathbf{x}, \mathbf{y}) \in R_{C}$ for all $\mathbf{y} \in S$.

Finally, we show that (2) is satisfied for $R=R_{C}$. Suppose $\mathbf{x}, \mathbf{y} \in X^{m}$ are such that $\left(x_{i} \mathbf{1}_{m}, y_{j} \mathbf{1}_{m}\right) \in R_{C}$ for all $i, j \in\{1, \ldots, m\}$. For $g \in\{1, \ldots, m\}$, let $\left(x_{i} \mathbf{1}_{m}\right)_{g}$ denote the $g^{\text {th }}$ component of the $m$-tuple $\left(x_{i}, \ldots, x_{i}\right)$. By definition of $R_{C}$, for each $i, j \in\{1, \ldots, m\}$, there are two possible cases:

(a) $\left(x_{i} \mathbf{1}_{m}, y_{j} \mathbf{1}_{m}\right) \in R_{C}^{d}$;

(b) $\left(\left(x_{i} \mathbf{1}_{m}\right)_{g},\left(y_{j} \mathbf{1}_{m}\right)_{h}\right) \in R_{C}^{d}$ for all $g, h \in\{1, \ldots, m\}$. 
Because $\left(x_{i} \mathbf{1}_{m}\right)_{g}=x_{i}$ and $\left(y_{j} \mathbf{1}_{m}\right)_{h}=y_{j}$ for all $g, h \in\{1, \ldots, m\},\left(x_{i} \mathbf{1}_{m}, y_{j} \mathbf{1}_{m}\right) \in$ $R_{C}^{d}$ follows in both cases. Thus, by definition of $R_{C}$, we obtain $(\mathbf{x}, \mathbf{y}) \in R_{C}$ and the proof is complete.

Note that the above proof does not make use of the assumption that $C$ is a choice function with a certainty inclusive domain; the conclusion of Theorem 1 remains true if $\Sigma$ can be any arbitrary non-empty domain. However, the certainty inclusiveness of $\Sigma$ is crucial for the results to be established in the following two sections.

\section{Transitive dominance rationalizability}

In traditional choice models that do not involve uncertainty, demanding rationalizability without any further restrictions on the rationalizing relation can be considered somewhat unsatisfactory. If, for instance, all rationalizations of a choice function generate strict preference cycles, it is difficult to think of the choice behavior thus revealed as coherent. The same reasoning applies to dominance rationalizability in the context of choosing from feasible sets of prospects: in addition to (1) and (2), one may want to demand that a dominance rationalization possesses some coherence property such as the well-established transitivity requirement. In this section, we show how transitivity can be incorporated into our model of choice under uncertainty. Interestingly, as mentioned at the end of the previous section, the certainty inclusiveness assumption on the domain of a choice function is important for the main result of this section.

Lemma 1 has a natural counterpart in the transitive setting (see Richter, 1971). All that needs to be done is to replace the relation $R_{C}$ with its transitive closure $t c\left(R_{C}\right)$ so that we obtain the following result.

Lemma 2 If a choice function $C$ is dominance rationalized by a transitive relation $R$, then $t c\left(R_{C}\right) \subseteq R$.

Proof Suppose that $R$ is a transitive dominance rationalization of $C$ and that $(\mathbf{x}, \mathbf{y}) \in$ $\operatorname{tc}\left(R_{C}\right)$. Thus, there exist $K \in \mathbb{N}$ and $\mathbf{x}^{0}, \ldots, \mathbf{x}^{K} \in X^{m}$ such that $\mathbf{x}=\mathbf{x}^{0},\left(\mathbf{x}^{k-1}, \mathbf{x}^{k}\right) \in$ $R_{C}$ for all $k \in\{1, \ldots, K\}$ and $\mathbf{x}^{K}=\mathbf{y}$. By Lemma 1 , it follows that $\mathbf{x}=\mathbf{x}^{0},\left(\mathbf{x}^{k-1}\right.$, $\left.\mathbf{x}^{k}\right) \in R$ for all $k \in\{1, \ldots, K\}$ and $\mathbf{x}^{K}=\mathbf{y}$. Because $R$ is transitive, we obtain $(\mathbf{x}, \mathbf{y}) \in R$.

Our characterization of dominance rationalizability by a transitive relation relies on the assumption that the domain of $C$ is certainty inclusive. Adapting direct dominance revelation coherence to the transitive framework considered in this section is straightforward and the requisite necessary and sufficient condition is obtained by replacing $R_{C}$ with its transitive closure $t c\left(R_{C}\right)$.

Transitive dominance revelation coherence. For all $S \in \Sigma$ and for all $\mathbf{x} \in X^{m}$,

$$
(\mathbf{x}, \mathbf{y}) \in t c\left(R_{C}\right) \text { for all } \mathbf{y} \in S \Rightarrow \mathbf{x} \in C(S)
$$

The main result of this section characterizes dominance rationalizability by a transitive relation. 
Theorem 2 A choice function $C$ is dominance rationalizable by a transitive relation if and only if $C$ satisfies transitive dominance revelation coherence.

Proof The proof of the only if part of the theorem is a straightforward adaptation of the proof of the only if part of Theorem 1; we leave it to the reader to verify that all that is required is to replace $R_{C}$ with $t c\left(R_{C}\right)$ and Lemma 1 with Lemma 2.

To prove the if part of the theorem, suppose that $C$ satisfies transitive dominance revelation coherence. We show that $R=t c\left(R_{C}\right)$ is a transitive dominance rationalization of $C$.

Clearly, $R=\operatorname{tc}\left(R_{C}\right)$ is transitive by definition.

To establish that (1) is satisfied for $R=t c\left(R_{C}\right)$, suppose first that $S \in \Sigma$ and $\mathbf{x} \in S$ are such that $(\mathbf{x}, \mathbf{y}) \in t c\left(R_{C}\right)$ for all $\mathbf{y} \in S$. By transitive dominance revelation coherence, $\mathbf{x} \in C(S)$.

Now suppose that $S \in \Sigma$ and $\mathbf{x} \in S$ are such that $\mathbf{x} \in C(S)$. By definition, this implies $(\mathbf{x}, \mathbf{y}) \in R_{C}^{d}$ for all $\mathbf{y} \in S$ and, because $R_{C}^{d} \subseteq R_{C} \subseteq t c\left(R_{C}\right)$, we obtain $(\mathbf{x}, \mathbf{y}) \in t c\left(R_{C}\right)$ for all $\mathbf{y} \in S$.

Finally, we show that (2) is satisfied for $R=t c\left(R_{C}\right)$. Suppose $\mathbf{x}, \mathbf{y} \in X^{m}$ are such that

$$
\left(x_{i} \mathbf{1}_{m}, y_{j} \mathbf{1}_{m}\right) \in t c\left(R_{C}\right) \text { for all } i, j \in\{1, \ldots, m\} .
$$

Because $\Sigma$ is a certainty inclusive domain, $\left\{x_{i} \mathbf{1}_{m}, y_{j} \mathbf{1}_{m}\right\} \in \Sigma$ for all $i, j \in\{1, \ldots, m\}$. Because $t c\left(R_{C}\right)$ dominance rationalizes $C$, (3) implies $x_{i} \mathbf{1}_{m} \in C\left(\left\{x_{i} \mathbf{1}_{m}, y_{j} \mathbf{1}_{m}\right\}\right)$ for all $i, j \in\{1, \ldots, m\}$. Thus, by definition of the direct revealed preference relation, we have $\left(x_{i} \mathbf{1}_{m}, y_{j} \mathbf{1}_{m}\right) \in R_{C}^{d}$ for all $i, j \in\{1, \ldots, m\}$. By definition of $R_{C}^{d}$, we obtain $(\mathbf{x}, \mathbf{y}) \in R_{C}$ and, because $R_{C} \subseteq t c\left(R_{C}\right)$, it follows that $(\mathbf{x}, \mathbf{y}) \in \operatorname{tc}\left(R_{C}\right)=R$.

\section{Suzumura consistent dominance rationalizability}

Full transitivity is often considered too demanding a requirement, especially in (but not restricted to) the context of collective choice. Thus, it is worthwhile to study the possibility of obtaining characterization results that employ notions of dominance rationalizability that are weaker than transitive dominance rationalizability and stronger than mere dominance rationalizability. One possibility to do so is to explore dominance rationalizability by a Suzumura consistent relation. The reason why we focus on Suzumura consistency as a suitable weakening of transitivity rather than on alternative properties such as quasi-transitivity or acyclicity is discussed in the concluding section of the paper.

As in the previous section, our starting point is an analogue of Lemma 1 where $R_{C}$ is replaced with its Suzumura consistent closure $\operatorname{sc}\left(R_{C}\right)$.

Lemma 3 If a choice function $C$ is dominance rationalized by a Suzumura consistent relation $R$, then $\operatorname{sc}\left(R_{C}\right) \subseteq R$.

Proof Suppose that $R$ is a Suzumura consistent dominance rationalization of $C$ and that $(\mathbf{x}, \mathbf{y}) \in \operatorname{sc}\left(R_{C}\right)$. By definition, we can distinguish two cases. 
(a) $(\mathbf{x}, \mathbf{y}) \in R_{C}$;

(b) $(\mathbf{x}, \mathbf{y}) \in \operatorname{tc}\left(R_{C}\right)$ and $(\mathbf{y}, \mathbf{x}) \in R_{C}$.

In case (a), Lemma 1 implies $(\mathbf{x}, \mathbf{y}) \in R$.

In case (b), there exist $K \in \mathbb{N}$ and $\mathbf{x}^{0}, \ldots, \mathbf{x}^{K} \in X^{m}$ such that $\mathbf{x}=\mathbf{x}^{0},\left(\mathbf{x}^{k-1}, \mathbf{x}^{k}\right) \in$ $R_{C}$ for all $k \in\{1, \ldots, K\}$ and $\mathbf{x}^{K}=\mathbf{y}$. Moreover, $(\mathbf{y}, \mathbf{x}) \in R_{C}$. By Lemma 1 , it follows that $\mathbf{x}=\mathbf{x}^{0},\left(\mathbf{x}^{k-1}, \mathbf{x}^{k}\right) \in R$ for all $k \in\{1, \ldots, K\}$ and $\mathbf{x}^{K}=\mathbf{y}$. Therefore, $(\mathbf{x}, \mathbf{y}) \in t c\left(R_{C}\right)$. Furthermore, $(\mathbf{y}, \mathbf{x}) \in R$ as a consequence of Lemma 1. If $(\mathbf{x}, \mathbf{y}) \notin R$, it follows that $(\mathbf{y}, \mathbf{x}) \in P(R)$. Because $(\mathbf{x}, \mathbf{y}) \in t c\left(R_{C}\right)$, this contradicts the Suzumura consistency of $R$. Thus, $(\mathbf{x}, \mathbf{y}) \in R$.

A necessary and sufficient condition for dominance rationalizability by a Suzumura consistent relation is obtained by employing the Suzumura consistent closure instead of the transitive closure when formulating the requisite coherence property.

Suzumura consistent dominance revelation coherence. For all $S \in \Sigma$ and for all $\mathbf{x} \in X^{m}$,

$$
(\mathbf{x}, \mathbf{y}) \in \operatorname{sc}\left(R_{C}\right) \text { for all } \mathbf{y} \in S \Rightarrow \mathbf{x} \in C(S) .
$$

The proof of our final characterization result is analogous to that of Theorem 2; we leave it to the reader to verify that all that is required is to replace the transitive closure with the Suzumura consistent closure and Lemma 2 with Lemma 3.

Theorem 3 A choice function $C$ is dominance rationalizable by a Suzumura consistent relation if and only if $C$ satisfies Suzumura consistent dominance revelation coherence.

\section{Concluding remarks}

In traditional models of rational choice on general domains without uncertainty, rationalizability by a transitive relation is equivalent to rationalizability by a reflexive, complete and transitive relation; see Richter (1966). The same observation applies to the current framework. Richter's (1966) proof technique employs a variant of Szpilrajn's (1930) extension theorem and proceeds by showing that any extension of the transitive closure of the direct revealed preference relation to a reflexive, complete and transitive relation also rationalizes $C$. That the dominance property does not change this result follows from the assumption that we operate on certainty inclusive domains. As a consequence of this property, the restriction of the direct revealed preference relation (and, thus, the restriction of $R_{C}$ ) to the set of certain prospects is already reflexive and complete. Therefore, no new pairs need to be added to the original relation as a consequence of the dominance requirement. However, the same argument does not apply to arbitrary domains because the above-mentioned reflexivity and completeness property of the restriction of $R_{C}$ is not guaranteed without assuming that $\Sigma$ is certainty inclusive. This means that there may exist extensions that do not obey the restrictions imposed by the definition of dominance rationalizability by a transitive relation, and existential clauses may have to be invoked to formulate necessary 
and sufficient conditions. See Bossert (2001) for analogous observations in the more restricted framework of set-based decision rules.

In contrast, Suzumura consistent dominance revelation coherence is not sufficient for dominance rationalizability by a reflexive, complete and Suzumura consistent relation. This is an immediate consequence of the observation that Suzumura consistency and transitivity coincide in the presence of reflexivity and completeness; see Suzumura (1976b).

The reason why we focus on Suzumura consistency as the weakening of transitivity to be considered is that properties such as quasi-transitivity or acyclicity cannot be treated in an analogous fashion. This is the case because there is no such thing as a quasi-transitive or an acyclical closure: if a relation fails to be quasi-transitive or acyclical, there is no unique way of defining a unique superset of this relation that possesses the requisite property. For instance, if $\mathbf{x}$ is strictly preferred to $\mathbf{y}, \mathbf{y}$ is strictly preferred to $\mathbf{z}$ and $\mathbf{z}$ is strictly preferred to $\mathbf{x}$, the resulting relation clearly is not acyclical (and, of course, not quasi-transitive). In order to obtain a superset of this relation that is acyclical, one of the pairs $(\mathbf{y}, \mathbf{x}),(\mathbf{z}, \mathbf{y})$ or $(\mathbf{x}, \mathbf{z})$ has to be added to the original relation, but any one of the three possibilities will do. Analogously, to obtain a quasi-transitive superset of the relation, two of the three pairs need to be added but, again, any two will do the job. Thus, there is no well-defined closure operation for these properties and, as a consequence, a condition that demands such a closure to be respected cannot be formulated. This observation also applies to dominance rationalizability by itself: because there does not exist a complete closure of a relation, our condition does not work if we want to obtain dominance rationalizability by a reflexive and complete relation. See Bossert and Suzumura (2010) for a detailed discussion of these issues in the traditional rational choice framework without uncertainty.

Our definition of the class of possible decision rules is very permissive - the dominance requirement appears to be quite uncontroversial. If one intends to come up with more restrictive notions of suitable decision models, the method suggested here may be applied to this alternative setting. Because of this observation, we think of this paper as providing two contributions: in addition to the results that we consider to be of interest in themselves, we propose a general method that can be employed when applying theories of rational choice to the analysis of decision making under uncertainty.

Open Access This article is distributed under the terms of the Creative Commons Attribution License which permits any use, distribution and reproduction in any medium, provided the original author(s) and source are credited.

\section{References}

Arrow KJ, Hurwicz L (1972) An optimality criterion for decision-making under ignorance. In: Carter CF, Ford JL (eds) Uncertainty and expectations in economics: essays in honour of G.L.S. Shackle. Basil Blackwell, Oxford, pp 1-11

Barberà S, Barrett CR, Pattanaik PK (1984) On some axioms for ranking sets of alternatives. J Econ Theory 33:301-308

Barberà S, Bossert W, Pattanaik PK (2004) Ranking sets of objects. In: Barberà S, Hammond P, Seidl C (eds) Handbook of utility theory, vol 2: extensions. Kluwer, Dordrecht, pp 893-977 
Barberà S, Pattanaik PK (1984) Extending an order on a set to the power set: some remarks on Kannai and Peleg's approach. J Econ Theory 32:185-191

Bossert W (2001) Choices, consequences, and rationality. Synthese 129:343-369

Bossert W, Sprumont Y, Suzumura K (2005) Consistent rationalizability. Economica 72:185-200

Bossert W, Suzumura K (2010) Consistency, choice, and rationality. Harvard University Press, Cambridge Fishburn PC (1970) Utility theory for decision making. Wiley, New York

Gärdenfors P (1976) Manipulation of social choice functions. J Econ Theory 13:217-228

Hansson B (1968) Choice structures and preference relations. Synthese 18:443-458

Houthakker HS (1950) Revealed preference and the utility function. Economica 17:159-174

Kannai Y, Peleg B (1984) A note on the extension of an order on a set to the power set. J Econ Theory 32:172-175

Kim KH, Roush FR (1980) Preferences on subsets. J Math Psychol 21:279-282

Milnor J (1954) Games against nature. In: Thrall R, Coombs C, Davis R (eds) Decision processes. Wiley, New York, pp 49-59

Richter MK (1966) Revealed preference theory. Econometrica 41:1075-1091

Richter MK (1971) Rational choice. In: Chipman JS, Hurwicz L, Richter MK, Sonnenschein HF (eds) Preferences, utility, and demand. Harcourt Brace Jovanovich, New York, pp 29-58

Samuelson PA (1938) A note on the pure theory of consumer's behaviour. Economica 5:61-71

Samuelson PA (1948) Consumption theory in terms of revealed preference. Economica 15:243-253

Savage LJ (1954) The foundations of statistics. Wiley, New York

Suzumura K (1976a) Rational choice and revealed preference. Rev Econ Stud 43:149-158

Suzumura K (1976b) Remarks on the theory of collective choice. Economica 43:381-390

Suzumura K (1977) Houthakker's axiom in the theory of rational choice. J Econ Theory 14:284-290

Szpilrajn E (1930) Sur 1'extension de 1'ordre partiel. Fundamenta Mathematicae 16:386-389

von Neumann J, Morgenstern O (1944) Theory of games and economic behavior. Princeton University Press, Princeton 